\title{
Survey of the initial management of celiac disease antibody tests by ordering physicians
}

\author{
Kathryn Potter ${ }^{1}$, Lawrence de Koning ${ }^{2,3}$, J. Decker Butzner ${ }^{1}$ and Dominica Gidrewicz ${ }^{1,4^{*}}$ (D)
}

\begin{abstract}
Background: Appropriate interpretation of a positive celiac antibody test by an ordering physician is important in order to institute proper management. We evaluated why children with an initial positive celiac serology were not referred for diagnostic biopsy or followed with serial testing by the ordering physician.

Methods: Consecutive celiac serologies in all patients less than 18 years of age were evaluated over 3.5 years and 775 children with a positive tissue transglutaminase antibody (TTG) were identified. If no management of a positive $\pi G$ could be identified, a survey was sent to the ordering physician. Responses were categorized as appropriate or inappropriate management.

Results: Of the 775 patients with a positive TT, 193 (24.9\%, 95\% Cl 21.9-28.1\%) received no follow-up management. We contacted 173 ordering physicians and 120 (69\%) responded. Of the 120 responses, 55 patients (45.8\%, 95\% Cl 36.8-55.1\%) were managed appropriately and 46 (38.3\%, 95\% Cl 29.7-47.7\%) were considered to be inappropriately managed when no repeat TTG was obtained within 18 months. Reasons for inappropriate management included: screen considered to be false positive (44.7\%), patient was not experiencing symptoms of celiac disease (31.6\%), symptoms had resolved (15.8\%), results were not indicative of celiac disease (26.3\%) and patients started a gluten-free diet with no evaluation of response (15.8\%). In 19 patients the TTG was not acted upon for technical reasons.
\end{abstract}

Conclusions: Positive TTGs require appropriate interventions. These include: subspecialist referral for further evaluation and/or repeat testing to evaluate: 1) treatment response or 2) patients with minimal or no symptoms.

Keywords: Celiac disease, Tissue transglutaminase, Pediatric, Diagnosis

\section{Background}

Celiac disease is an autoimmune enteropathy triggered by ingested gluten in genetically susceptible individuals, and affects approximately $1 \%$ of the population $[1,2]$. Timely and accurate diagnosis of celiac disease is limited because physicians do not recognize atypical presentations, including chronic abdominal pain or chronic constipation [3, 4]. In addition approximately $60 \%$ of children present with extra-intestinal manifestations of celiac disease including fatigue, headaches, growth

\footnotetext{
* Correspondence: Dominica.gidrewicz@ahs.ca; dominica.gidrewicz@ahs.ca ${ }^{1}$ Department of Pediatrics, Cumming School of Medicine, University of Calgary, Calgary, Canada

${ }^{4}$ Division of Pediatric Gastroenterology, Alberta Children's Hospital, 2888

Shaganappi Trail, Calgary, AB T3B 6A8, Canada

Full list of author information is available at the end of the article
}

failure, arthralgias, and iron deficiency anemia $[3,5,6]$. Many physicians are not aware of these atypical presentations of celiac disease that result in delays in diagnosis $[7,8]$. Two-thirds of patients consult at least two physicians prior to the diagnosis [8]. Delayed diagnosis and persistent intestinal inflammation lead to complications that include persistent gastrointestinal symptoms, pubertal delay, osteoporosis, autoimmune disorders, and increased risk of non-Hodgkin lymphoma [9-13]. An economic evaluation demonstrated that in the year prediagnosis, persistent health concerns were investigated at an estimated cost of $\$ 8500$ US per capita [14]. Delayed diagnosis taxes both the individual and the health care system.

(c) The Author(s). 2019 Open Access This article is distributed under the terms of the Creative Commons Attribution 4.0 International License (http://creativecommons.org/licenses/by/4.0/), which permits unrestricted use, distribution, and reproduction in any medium, provided you give appropriate credit to the original author(s) and the source, provide a link to the Creative Commons license, and indicate if changes were made. The Creative Commons Public Domain Dedication waiver (http://creativecommons.org/publicdomain/zero/1.0/) applies to the data made available in this article, unless otherwise stated. 
Celiac disease antibody screening with the tissue transglutaminase (TTG) antibody test identifies individuals who require further testing to diagnose celiac disease or alterations in management of those with known celiac disease. Management decisions following a positive celiac screen are influenced by the degree of elevation of the TTG and severity of patient symptoms. In addition, the TTG may be falsely positive in individuals with minimal or no symptoms such as first-degree relatives of patients with celiac disease or individuals who have medical conditions associated with celiac disease [4]. Unfortunately other autoimmune diseases and intercurrent infections can cause false positive TTG results and must be considered when managing patients with minimal symptoms [15]. Thus, many factors influence how celiac serology should be interpreted. "Choosing Wisely" is an initiative to help clinicians to appropriately manage abnormal test results and reduce unnecessary testing [16]. Physicians must understand when a test should be ordered and how to interpret the result. By identifying general errors in management, physicians can improve patient care and reduce health care costs.

Appropriate management of children with possible celiac disease begins with correct identification of those children that require further testing and/or subspecialty evaluation. We hypothesized that a proportion of children who do not undergo an intestinal biopsy did not receive further evaluation of an initial positive celiac screen and/or started a gluten-free diet without ongoing monitoring of their celiac serology. Thus, the aim of our study was to evaluate the management of an initial positive celiac screening test by the ordering physician in order to understand how physicians manage celiac serology test results. The data were used to develop educational strategies that address these issues in order to decrease the time to diagnosis and improve quality of care for children with celiac disease.

\section{Methods}

Consecutive patients $(<18$ years of age) with celiac serology were identified in the Calgary Laboratory Services (CLS) database over 3.5 years from July 2008 to December 2011. All patients had a serum IgA and IgA-tissue transglutaminase (TTG) performed (Euroimmune, Germany). If the TTG was positive (>20 kU/L), an IgA anti-endomysial antibody (EMA) test was measured (IMMCO Diagnostics, NY). Intestinal biopsy results were obtained from the Alberta Children's Hospital pathology database. This hospital is the only pediatric gastroenterology referral center in southern Alberta, Canada, that provides service to a large pediatric population of over 460,000 children. It is staffed by nine pediatric gastroenterologists.
The electronic medical record, clinic charts and small intestinal pathology reports were obtained on all patients with a positive TTG. Those patients with no record of intestinal biopsy or consultation with a pediatric gastroenterologist were identified for the survey study. A questionnaire (Additional file 1: Figure S1) was mailed to the ordering physician to evaluate the management of these TTG-positive patients.

Patients were classified into three groups: high TTG ( $\geq$ $10 \mathrm{x}$ the upper limit of normal, ULN, $\geq 200 \mathrm{kU} / \mathrm{L}$ ), moderately elevated TTG (3-10 x ULN, 60-199 kU/L), and low TTG ( $<3 \times$ ULN, 20-59 kU/L). These cut-offs corresponded to those set out in the ESPGHAN guidelines on the diagnosis of celiac disease in children $[4,17]$. Physician management in this study occurred prior to the publication of these guidelines. Management was considered appropriate if patients with a TTG $\geq 3 \times$ ULN were referred for evaluation by a gastroenterologist. Patients with minimal or no symptoms and a TTG of $<3 \mathrm{x}$ ULN could be referred to a pediatric gastroenterologist or followed with subsequent celiac antibody levels to evaluate if they decreased $[4,17]$. Patients with a TTG < $3 \times$ ULN and negative EMA have only a $13 \%$ positive predictive value of biopsy proven celiac disease [17]. Inappropriate management was assigned to patients with a TTG $\geq 3 \times$ ULN ( $\geq 60 \mathrm{kU} / \mathrm{L})$ who were not referred and did not have repeat serology within 18 months of the first celiac screen. Patients with a TTG $\geq 10 \times$ ULN and positive EMA (who may qualify for a non-biopsy diagnosis under ESPGHAN criteria) were assigned to the inappropriate management category if no repeat TTG was performed.

Where appropriate, mean, median, significant difference between proportions and $95 \%$ confidence intervals (CIs) were calculated. The study was approved by the University of Calgary Medical Ethics Conjoint Health Research Ethics Board.

\section{Results}

Of the 17,505 children who had celiac serology performed, 775 patients had a positive TTG [17]. Of these, 543 (70\%; 95\% CI 66.7-73.2\%) were referred to the pediatric gastroenterology service at the Alberta Children's Hospital, 39 (5.0\%; 95\% CI 3.6-6.9\%) with known celiac disease had repeat serology, and 193 patients (24.9\%; 95\% CI 21.9-28.1\%) with a positive TTG were not referred for pediatric gastroenterology evaluation. Of the 193, the ordering physician was not identified in 20 patients. Thus 173 surveys were distributed and 120 were completed and returned. The study response rate was $69.4 \%$, composed of family practitioners $(57.8 \%)$, pediatricians/pediatric subspecialists (38.5\%) and internal medicine subspecialists (3.6\%). Of the pediatricians, 
$59.3 \%$ were community pediatricians, $12.5 \%$ hospitalists, and $28.2 \%$ pediatric subspecialists. Of the 775 patients with a positive TTG, we could not evaluate management in $73(9.4 \%$, Table 1$)$.

Of the 120 patients with completed questionnaires, 55 (45.8\%, 95\% CI 36.8-55.1\%) patients were considered to be managed appropriately. This included either a repeat TTG within 18 months of the previously elevated TTG and/or referral for subspecialty evaluation for further management (Table 1, Additional file 2: Figure S2). All of the nine patients with a TTG $>3 \times$ ULN and positive EMA were appropriately managed within four months (Additional file 2: Figure S2). Of those with a moderately elevated TTG (3-10 x ULN), 8/15 were determined likely a false positive and the TTG was repeated a median 3.5 months later (range 2.5 to 15 months). The TTG returned to normal in six patients or was only 1.5 $x$ ULN in two. Of the 36 with a low TTG $(<3 x$ ULN), four had known celiac disease, six were referred for subspecialty evaluation and 26 patients had a repeat TTG a median of 6 months later (range 1-18 months). In 18 patients, the TTG had normalized upon repeat testing.

Of the 120 patients, 46 (38.3\%, 95\% CI 29.7-47.7\%) were managed inappropriately including 18 children who had a repeat TTG at least 3.5 years after the initial TTG and 28 children with no repeat TTG (Table 1). All five of the patients with a TTG $\geq 10 \mathrm{x}$ ULN started a GFD without appropriate follow-up testing to assess response to a GFD (four patients had no repeat TTG, one had a repeat TTG over 6 years later). Of the 10 patients with a moderately elevated TTG (3-10 x ULN), only five had a repeat TTG (median 38 months later, range 2150 months), while five had no repeat TTG and the ordering physician did not feel the test was indicative of celiac disease based on the patient's symptoms. Two-thirds of inappropriately managed patients (31/ 46) had a low TTG $(<3 \times$ ULN). Only 12 had a repeat TTG (median 37 months, range $25-83$ months), of which four patients had an increased TTG upon repeat testing. Nine patients who refused GI evaluation did not have a repeat TTG.

As physicians may act more cautiously with younger patients, we evaluated if the patient's age at initial TTG influenced a physician's follow-up management. We characterized the appropriately and inappropriately managed patients into two groups: under six years of age $(n=26)$ and greater than six years $(n=75)$. Of the children less than six, 12/26 (46.1\%; 95\% CI 28.7-64.5\%) were inappropriately managed, as compared to $34 / 75$ (45.3\%; 95\% CI $34.6-56.6 \%$ ) of those over six, thus showing a similar proportion of inappropriately managed patients in both groups.

The TTG was not acted upon for technical reasons in 19 patients $(15.8 \%, 95 \%$ CI $10.0-23.9 \%)$ (Table 1$)$. The TTG was classified as a technical error when the ordering physician: was unable to contact the patient or the patient moved away (6), did not order the test (10), did not receive the results (1), or patient records were no longer available (2).

The most common reasons for not referring for gastrointestinal evaluation included: the screen was considered a false positive (51.2\%), the patient was not experiencing symptoms of celiac disease (39.5\%), or the results did not appear indicative of celiac disease (30.2\%) (Table 2).

Sixty-five physicians listed sources of information for the diagnosis and management of celiac disease. The majority of respondents derive their information from formal continuing medical education, including medical journals (34\%), conferences (25\%), Internet materials $(22 \%)$ and guidelines $(8 \%)$. Other sources included expert opinion (46\%), residency training (18\%) and other $(9 \%)$.

\section{Discussion}

This observational survey evaluated management decisions of celiac disease antibody tests by ordering physicians. As the first point of contact for patients, the

Table 1 Summary of patients in whom follow-up management was evaluated ( $n=193$ )

\begin{tabular}{lllll}
\hline & Appropriate $(n=55)$ & Inappropriate $(n=46)$ & Technical $(\mathrm{n}=19)$ & Nonresponders $(n=73)$ \\
\hline Mean age at initial TTG (yrs) & $9.9+/-5.2$ & $9.1+/-4.8$ & $9.9+/-5.0$ & $9.2+/-5.25$ \\
Female, $\mathrm{n}(\%)$ & $27(49.1)$ & $23(50.0)$ & $13(68.4)$ & $49(67.1)$ \\
$\begin{array}{l}\text { EMA positive, } \mathrm{n}(\%) \\
\text { TTG, } \mathrm{n}(\%)\end{array}$ & $10(16.7)$ & $7(15.2)$ & $8(42)$ & $19(26)$ \\
$\quad \geq 10 \times$ ULN & & & \\
$3-10 \times$ ULN & $4(7.3)$ & $5(10.9)$ & $6(31.6)$ & $17(23.3)$ \\
$1-3 \times$ ULN & $15(27.3)$ & $10(21.7)$ & $2(10.5)$ & $14(19.2)$ \\
\hline
\end{tabular}

$E M A$ anti-endomysial antibody

$\pi G$ tissue transglutaminase antibody

ULN upper limit of normal

The only statistical difference was between the appropriate \& inappropriately managed patients with a $\Pi G \geq 10 \times$ ULN (9/101) versus the nonresponders with a $\pi \mathrm{TG} \geq 10 \times$ ULN (17/73) $(p<0.05)$ 
Table 2 Reason(s) for not referring for gastrointestinal evaluation ( $\mathrm{n}=$ respondents) $\mathrm{n}$ (\%)

\begin{tabular}{lll}
\hline & Appropriate $(n=33)$ & Inappropriate $(n=38)$ \\
\hline Low antibody titre likely a false positive & $17(51.5)$ & $17(44.7)$ \\
Patient was not experiencing symptoms of CD & $13(39.4)$ & $12(31.6)$ \\
Results did not appear to be indicative of CD & $10(30.3)$ & $10(26.3)$ \\
Patient unwilling to undergo intestinal biopsy & $5(15.2)$ & $8(21.0)$ \\
Symptoms had resolved & $3(9.1)$ & $6(15.8)$ \\
Patient already on a gluten-free diet & $2(6.1)$ & $6(15.8)$ \\
False positive due to a known medical condition & $8(24.2)$ & $3(7.9)$ \\
\hline
\end{tabular}

rationale behind proper physician management of a positive celiac screen has important implications for the patients. Of the patients with a positive celiac antibody test who were not referred to a gastroenterologist, 38\% were managed inappropriately. The frequency of inappropriate management of patients could rise to as high as $71 \%$ if all survey "technical no contact" and "non-responder" individuals are included in that group.

We identified multiple reasons why physicians inappropriately managed celiac disease antibody tests. Of the patients with a low titer TTG $(<3 \times$ ULN) and inappropriate management, physicians indicated that the initial screen was indicative of a false positive such as due to lack of symptoms or symptoms had resolved. However, in order to properly diagnose a false positive test in these situations, a patient must have a repeat TTG that normalizes while consuming gluten. A low positive TTG may indicate early celiac disease. In fact, $19 \%$ of the patients who had a repeat TTG over three years later, displayed an increased TTG and experienced a delayed diagnosis of celiac disease. Additional erroneous assumptions that led to inappropriate management included: no referral because symptoms resolved, starting on a GFD with no follow-up testing to evaluate response to a gluten free diet, and patient refusal for further evaluation. Families may lack the understanding to differentiate between a screening test result with the potential for a false positive result and a gold-standard diagnostic test, such as an intestinal biopsy. In addition, children with minimal or no symptoms and a weakly positive antibody test may develop celiac disease with higher antibody levels over time [4]. Repeat antibody testing is required to evaluate these clinical scenarios.

For the appropriate interpretation and management of a positive screening test, one must stratify patients into low-risk patients who are unlikely to have celiac disease, and identify high-risk patients who require an intestinal biopsy to confirm the diagnosis and to evaluate response to a GFD $[4,9,17]$. Although there is growing awareness of celiac disease, adults without gastrointestinal symptoms have a mean delay in diagnosis of 42 months [7].
Long delays in diagnosis contribute to a negative impact on patient quality of life and increase health utilization and costs [14]. This study reflects the goals of the "Choosing Wisely" campaign that seeks to encourage physicians to make effective choices to ensure high quality care [16]. In order to improve how physicians choose to order, interpret, and follow celiac serology tests, one must first identify incorrect assumptions in laboratory test interpretation and subsequently develop educational tools to improve primary care physician management decisions.

Given the large proportion of asymptomatic patients with a positive TTG on a gluten containing diet who are at risk of celiac disease complications, a positive celiac screen needs to be serially monitored. If the initial TTG is positive but low $(<3 \mathrm{x} U \mathrm{ULN})$ with a negative EMA, then a false-positive result is likely $[4,17]$. In the absence of any signs or symptoms, the ordering physician may follow the child on a gluten-containing diet and serological testing repeated every 6-12 months until the antibody levels normalize or increase to a level where biopsy is indicated. If the EMA is positive, then the likelihood for celiac disease increases because of the high specificity of EMA, and such patients, even at low titer TTGs, should be referred for specialist evaluation and endoscopy $[4,17]$.

The clinical presentation of a patient influences a physician's interpretation of the initial TTG. Though a physician may choose to act more cautiously in younger children, our study did not show that children over six years of age were more likely inappropriately managed compared to younger children. Atypical presentations, however, are increasingly common in children [4-6], such that close to $60 \%$ of children may be asymptomatic or present atypically [5]. A common physician error was the assumption that a patient with a positive screening test was less likely to have celiac disease if the child lacked classic symptoms. A large study identified seven new cases of celiac disease presenting atypically in children for each new case of celiac disease that presented with the classical symptoms of celiac disease [18]. 
Therefore, current pediatric and adult guidelines recommend testing for celiac disease in a large variety of ailments, including nonspecific gastrointestinal symptoms such as chronic abdominal pain or IBS, and non-GI (atypical) symptoms such as nutritional anemia, chronic fatigue, short stature, delayed puberty, dental enamel defects, elevated liver transaminases levels and dermatitis herpetiformis $[4,11]$.

Furthermore, $15 \%$ of patients without appropriate follow-up serial TTG started a gluten-free diet prior to diagnosis against medical advice. It is important for medical practitioners to emphasize that a confirmed diagnosis is essential to justify starting a gluten-free diet that must be followed for life. This diet is expensive, complicated and difficult to manage, and therefore patients with newly diagnosed celiac disease should be strongly encouraged to meet with a dietitian skilled in the gluten-free diet to prevent dietary contamination and assist with long-term adherence [4, 9].

This study provides valuable information as it includes a comprehensive number of physicians and their management of elevated celiac screen. The study has limitations including the retrospective data collection and recall bias as the surveys were mailed out up to 4 years after the original TTG was ordered. We were unable to verify that all TTG screens were done on patients who consumed gluten. In those who underwent repeat serology, we could not assess dietary compliance. A response rate of $68 \%$ is acceptable but not complete.

\section{Conclusions}

This observational study demonstrates common errors in celiac serology interpretation by ordering physicians that include failure to: follow up low titres, recognize that celiac disease may occur in patients without classic celiac symptoms, and evaluate TTG serologies of patients who decline duodenal biopsy, including those who start a gluten-free diet. Physicians need to be educated about the risks of undetected celiac disease in asymptomatic or mildly symptomatic patients and the higher incidence of celiac disease in autoimmune conditions that can cause false positive screening tests. Positive TTGs, even $<3 \times$ ULN, need follow up within a year, and if titres increase a consultation for further evaluation. TTGs $\geq 3 \times$ ULN should be referred for consultation for intestinal biopsy and those diagnosed with celiac disease need periodic repeat TTGs to evaluate response to a gluten-free diet. Improvement in the management of positive celiac disease screening tests will lead to early disease identification, minimize unnecessary testing for other conditions, improve patient's quality of life and reduce healthcare costs.

\section{Additional file}

Additional file 1: Figure S1. Study questionnaire. (PDF $124 \mathrm{~kb}$ )

Additional file 2: Figure S2. Study flow diagram. (PPTX $32 \mathrm{~kb}$ )

\section{Abbreviations}

Cl: Confidence Interval; EMA: Anti-endomysial antibody; GFD: Gluten-free diet; TTG: Tissue transglutaminase; ULN: Upper limit of normal

\section{Acknowledgements \\ The Canadian Celiac Association recently developed educational tools to assist physicians in the diagnosis and management of celiac disease. They are found in the "Healthcare Professionals" section at (www.celiac.ca).}

\section{Authors' contributions}

DG and JDB conceptualized and designed the study. LDK acquired the TTG results from the Calgary Lab Services database. KP, DG, JDB designed the questionnaire survey, and KP mailed the survey, tabulated the survey results, carried out the statistics and drafted the initial manuscript. DG, KP, LDK and JDB approved the final manuscript as submitted.

\section{Funding}

The study was supported by grants from the Canadian Celiac Association, Calgary Chapter. None of the funders had any influence on this study.

\section{Availability of data and materials}

The datasets used and/or analysed during the current study are available from the corresponding author on reasonable request.

\section{Ethics approval and consent to participate}

The study was approved by the University of Calgary Medical Ethics Conjoint Health Research Ethics Board. The study participants were the ordering primary care physician and Calgary Laboratory Services (CLS). Ethics approval was obtained to review the CLS data and to contact the ordering physicians about patient management. The celiac screen was obtained for clinical indications by the ordering physician. No additional blood work was requested of the patient for this study. Consent was presumed by the ordering physicians who completed and returned the survey.

Consent for publication

Not applicable.

\section{Competing interests}

The authors declare that they have no competing interests.

\section{Author details}

'Department of Pediatrics, Cumming School of Medicine, University of Calgary, Calgary, Canada. ${ }^{2}$ Department of Pathology and Laboratory Medicine, Cumming School of Medicine, University of Calgary, Calgary, Canada. ${ }^{3}$ Calgary Laboratory Services Calgary, Calgary, Alberta, Canada. ${ }^{4}$ Division of Pediatric Gastroenterology, Alberta Children's Hospital, 2888 Shaganappi Trail, Calgary, AB T3B 6A8, Canada.

Received: 30 December 2018 Accepted: 10 July 2019 Published online: 19 July 2019

\section{References}

1. Hoffenberg EJ, MacKenzie T, Barriga KJ, Eisenbarth GS, Bao F, Haas JE, et al. A prospective study of the incidence of childhood celiac disease. J Pediatr. 2003;143:308-14.

2. Fasano A, Berti I, Gerarduzzi T, Not T, Colletti RB, Drago S, et al. Prevalence of celiac disease in at-risk and not-at-risk groups in the United States: a large multicenter study. Arch Intern Med. 2003;163:286-92.

3. McGowan KE, Castiglione DA, Butzner JD. The changing face of childhood celiac disease in North America: impact of serological testing. Pediatrics. 2009;124:1572-8.

4. Husby S, Koletzko S, Korponay-Szabo IR, Mearin ML, Phillips A, Shamir R, et al. European Society for Pediatric Gastroenterology, Hepatology, and Nutrition guidelines for the diagnosis of coeliac disease. J Pediatr Gastroenterol Nutr. 2012;54:136-60. 
5. Jericho H, Sansotta N, Guandalini S. Extraintestinal manifestations of celiac disease: effectiveness of the gluten-free diet. J Pediatr Gastroenterol Nutr. 2017;65:75-9.

6. Leffler DA, Green PH, Fasano A. Extraintestinal manifestations of coeliac disease. Nat Rev Gastroenterol Hepatol. 2015;12:561-71.

7. Paez MA, Gramelspacher AM, Sinacore J, Winterfield L, Venu M. Delay in diagnosis of celiac disease in patients without gastrointestinal complaints. Am J Med. 2017;130:1318-23.

8. Cranney A, Zarkadas M, Graham ID, Butzner JD, Rashid M, Warren R, et al. The Canadian celiac health survey. Dig Dis Sci. 2007;52:1087-95.

9. Snyder J, Butzner JD, DeFelice AR, Fasano A, Guandalini S, Liu E, et al. Evidence-informed expert recommendations for the management of celiac disease in children. Pediatrics. 2016;138(3).

10. Cosnes J, Cellier C, Viola S, Colombel JF, et al. Incidence of autoimmune disease in celiac disease: protective effect of the gluten-free diet. Clin Gastroenterol Hepatol. 2008;6:753-8.

11. Rubio-Tapia A, Hill ID, Kelly CP, Calderwood AH, Murray JA. ACG clinical guidelines: diagnosis and management of celiac disease. Am J Gastroenterol. 2013;108:656-76.

12. Fouda MA, Khan AA, Sultan M, Pios LP, McAssey K, Armstrong D. Evaluation and management of skeletal health in celiac disease: position statement. Can J Gastroenterolol. 2012:26:819-29.

13. Tio M, Cox MR, Eslick GD. Meta-analysis: coeliac disease and the risk of allcause mortality, any malignancy and lymphoid malignancy. Aliment Pharmacol Ther. 2012;35:540-51.

14. Green PH, Neugut Al, Naiyer AJ, Edwards ZC, Gabinelle S, Chinburapa V. Economic benefits of increased diagnosis of celiac disease in a national managed care population in the United States. J Insur Med. 2008;40:218-28.

15. Hill I. Guideline for the diagnosis and treatment of celiac disease in children: Recommendations of the North American Society for Pediatric Gastroenterology, Hepatology and Nutrition. J Pediatr Gastroenterol Nutr. 2005:40:1-19.

16. Wintemute K, McDonald K, Huynh T, Pendrith C, Wilson L. Addressing overuse starts with physicians: Choosing Wisely Canada. Can Fam Physician. 2016;62:199-200, 207-9.

17. Gidrewicz D, Potter K, Trevenen CL, Lyon M, Butzner JD. Evaluation of the ESPGHAN celiac guidelines in a North American pediatric population. Am 」 Gastroenterol. 2015;110:760-7.

18. Catassi C, Fabiani E, Ratsch IM, Coppa GV, Giorgi PL, Pierdomenico R, et al. The coeliac iceberg in Italy. A multicentre antigliadin antibodies screening for coeliac disease in school-age subjects. Acta Paediatr 1996; 412 Suppl:29-35.

\section{Publisher's Note}

Springer Nature remains neutral with regard to jurisdictional claims in published maps and institutional affiliations.

Ready to submit your research? Choose BMC and benefit from:

- fast, convenient online submission

- thorough peer review by experienced researchers in your field

- rapid publication on acceptance

- support for research data, including large and complex data types

- gold Open Access which fosters wider collaboration and increased citations

- maximum visibility for your research: over $100 \mathrm{M}$ website views per year

At $\mathrm{BMC}$, research is always in progress.

Learn more biomedcentral.com/submissions 